Ritter, C., Zhang, Y., Dupuis, R., and Al-Hussein, M. (2017). "Simulation of Production Line Improvement in Modular Home Manufacturing." In: LC3 2017 Volume II - Proceedings of the 25th Annual Conference of the International Group for Lean Construction (IGLC), Walsh, K., Sacks, R., Brilakis, I. (eds.), Heraklion, Greece, pp. 737-744. DOI: https://doi.org/10.24928/2017/0232

\title{
SIMULATION OF PRODUCTION LINE IMPROVEMENT IN MODULAR HOME MANUFACTURING
}

\author{
Chelsea Ritter ${ }^{1}$, Youyi Zhang $^{2}$, Roger Dupuis ${ }^{3}$, and Mohamed Al-Hussein ${ }^{4}$
}

\begin{abstract}
The production of modular homes is highly variable, where the production time for a single home can vary significantly. Because there is a large amount of variation and customization in the home models built, operation time on the production line varies greatly, and considerable effort is needed for production line flow balancing. Simulation not only allows the manufacturer to identify areas of potential improvement on the line, but also helps to measure the likely success of proposed changes before investment in or implementation of these changes. In this paper, a case study is presented which uses simulation to evaluate the current-state performance of a wall panel production line, and to validate several proposed changes in terms of whether or not they will be effective and should be implemented.
\end{abstract}

Keywords: Production line performance, Simulation, Lean, Modular home manufacturing.

\section{INTRODUCTION}

Modular home construction plays a unique role in today's construction industry due to the associated potential for continuous improvement with respect to product innovation, quality, and efficiency (Moghadam et al., 2012). Modular manufacturers are continuously seeking ways to enhance their operations through the application of Lean manufacturing techniques; research and development $(\mathrm{R} \& \mathrm{D})$ teams bring out new ideas to challenge the current practice. Testing and implementation plays a key role in establishing a culture of acceptance of new ideas and ensuring a successful Lean manufacturing transformation journey. However, the unknowns of any proposed methodology constitute a barrier to implementation because of the extra cost of the test run, as well as the potential for failure of newly adopted work procedures after a large capital investment. In this regard, simulation has proven to be effective in filling the gap between $R \& D$ and production line operation during the improvement process.

This paper investigates the use of simulation to model a wall panel fabrication line in a modular home manufacturing facility in order to assist management in identifying potential areas of improvement as well as to provide strategies for implementation of the proposed changes. A future-state simulation model is presented which can generate a preliminary validation report on the feasibility of the proposed methodology.

MSc Student, Faculty of Civil and Environmental Engineering, Hole School of Construction Engineering, University of Alberta, Edmonton, Alberta, Canada, critter1@ualberta.ca

2 MSc Student, Faculty of Civil and Environmental Engineering, Hole School of Construction Engineering, University of Alberta, Edmonton, Alberta, Canada, youyi@ualberta.ca

3 Safety - Quality - Continuous Improvement Manager, Kent Homes, Bouctouche, New Brunswick, Canada, dupuis.roger@kenthomes.com

4 Professor, Faculty of Civil and Environmental Engineering, Hole School of Construction Engineering, University of Alberta, Edmonton, Alberta, Canada, malhussein@ualberta.ca 


\section{LITERATURE REVIEW}

The potential for the use of simulation in modular home production lines has been recognized and tested in other studies. Shafai (2012) notes the importance of avoiding overproduction, where using a pull system, establishing continuous flow, and eliminating waste are recognized as some of the salient Lean concepts to be leveraged. Because of the variability in the design and requirements for each house, relying on average task times for activities with high amounts of variation is found to be inaccurate, and instead the use of statistical analysis to develop a distribution for each task time is recommended. For highly variable tasks, Shafai (2012) notes that design properties influence the task duration. For walls, design properties may include the number of window and door openings, the length, and the number of studs. Regression analysis is typically applied to determine the statistically relevant design properties, and to find regression models for highly variable activities.

Moghadam (2014) also applies simulation to their study of modular construction manufacturing process improvement. They use simulation to evaluate the labour required in a modular construction manufacturing facility to meet a range of different takt time goals. Labour resources are categorized based on whether they have been crosstrained to work at different workstations or are only qualified to work at a certain workstation. In the research presented in this paper, while simulation is used to evaluate labour in relation to takt time, it is also used to evaluate semi-automated machinery.

$\mathrm{Yu}$ et al. (2013) assert that the first step in creating standardized work is to determine the takt time, which will set the pace for the production of the entire line. The takt time is the maximum time spent at one station, and can be calculated as the time available divided by the demand. They also note that the time required for a single task can vary drastically, pointing out that the use of a labour resource pool could help to decrease this variability.

The use of simulation for manufacturing as a general industry is also supported by McLean and Leong (2001), who support the position that while currently underutilized, simulation in manufacturing can help to decrease start-to-finish production time and increase the quality of the products produced. They mention that costs, time, and skill of employees are some of the reasons that simulation may not be currently used in these environments.

\section{METHODOLOGY}

In order to build a reliable and effective simulation model, site observation and time study are essential. Therefore, a study of the current work sequence is first carried out. Understanding the procedure is crucial for ensuring the accuracy of the data collection and evaluation. The level of accuracy of the data can vary based on the type of task being studied. It is thus important to design the data collection process based on the purpose of the output. In this case, the method used to understand the problem and the current state of production is to interview an expert on the given company's operations.

Next, the simulation model is built based on the collected information. The building of the current-state simulation model involves defining the targeted area for the simulation; constructing the simulation model; analyzing the model and its output; and determining potential areas of process improvement. The Cyclone template in Simphony is used to create the simulation model representing the current state, and then to apply any proposed changes in order to quantify the effects with respect to several different 
metrics of the wall line's success, such as productivity and labour utilization. Simphony, developed at the University of Alberta, is "an integrated environment for building special purpose simulation tools for modelling construction systems" (AbouRizk and Mohamed, 2001). Cyclone is a general purpose template provided in Simphony that is especially useful for modelling continuous or cyclical events. The discrete modelling function in Simphony is used in this study to model the production line. Finally, the proposed improvements are tested by changing the simulation model accordingly and using the results to quantify the effects of the change.

\section{CASE STUdY}

\subsection{Data Collection}

Two different types of modular homes are constructed in the case facility-mini homes and modular homes-each of which has a range of sizes and many custom options that can be applied. Each mini home is a self-contained module, while the modular homes each consist of two modules that are later attached to one another on site.

In this case, it is assumed that data collection based on expert opinions will be accurate enough to determine the expected improvements based on the identified case changes. A person familiar with the plant activities is surveyed in order to determine a lower bound, upper bound, and most likely duration for the various activities as well as the current plant output for use in constructing and verifying the model. Because of this data collection strategy, triangular distributions are used in constructing the model. Using this data, the under-utilized wall production line is studied in detail through the use of simulation in order to test different proposed improvements and identify their effectiveness in increasing the production to a target of walls for four houses produced per day. Simulation allows the effects of any changes to the wall production line to be tested and analysed without disrupting the line or investing in costly equipment.

\subsection{Current State Simulation Model}

\subsubsection{Side Lumber Construction Station}

The side lumber station is one of the first stations on the line and is where the construction of the side lumber, referred to as the top and bottom plates, is done. This step involves attaching $16 \mathrm{ft}$ lengths of lumber to reach the total desired length of the module. The assembly of these lumber pieces needs to be completed upstream of the construction of the framed exterior wall.

\subsubsection{Openings Construction Station}

This station is where the wall openings for doors and windows are constructed for the exterior wall panels. The number of openings per wall and the type and size of openings will vary based on the home type and the customer's preferences. The time required to construct each opening is based on the type of opening, (e.g., door, or small, medium, or large window). Triangular distributions are used to predict the durations. As is the case for the top and bottom sills, the construction of the openings will need to be completed before the construction of the exterior wall panel can be done.

\subsubsection{Exterior Wall Panel Construction Station}

Once the required openings and the top and bottom sill for the exterior wall panel have been constructed, the panel can be put together. This process involves installing studs 
and the components for the openings. The simulation model will calculate how many studs and opening components are required and use these characteristics to calculate the time required to construct the wall. The fastening of the studs to the top and bottom plates is assumed to have a constant time but the total construction time for the wall will vary based on how many studs and opening components are in the wall.

\subsubsection{Interior Wall Framing Station}

The interior wall framing is carried out in the same manner as for the exterior walls, but they are usually significantly smaller. The time required for fabrication of each interior wall is calculated as the time required to attach each stud to the top and bottom plates, multiplied by the number of studs required for each wall. The number and size of interior walls will be determined by the model and size of the home, and will vary based on the customer's order.

\subsubsection{Non-Conventional Interior Wall Framing Station}

Irregularly shaped interior walls are included only in modular home models and include walls with curves, half-walls, or other shapes that cannot be produced at the same rate as regular interior walls. Therefore, the time required to construct these panels is highly variable. A triangular distribution is thus used to predict the time needed for the construction of each non-conventional wall.

\subsubsection{Poly and Drywall Installation on Exterior Walls Station}

At this station, the poly for the exterior walls is installed and, if time permits, some drywall is installed as well. As a general rule, it is assumed that drywall will only be installed until the other two parallel stations (the special shape wall construction and the interior wall framing) have completed their work for the given unit being produced, so that the wall erection will not delayed.

\subsubsection{Labour Assignment}

There are 7 workers assigned to the wall manufacturing line. The current-state labour requirement of each station can be seen in Table 4-1. As can be seen, the sum of the labour requirements is greater than the amount of workers. This is because not all the stations are fully utilized, so workers can be shared between stations.

Table 4-1: Labour resources per station in the current state

\begin{tabular}{cccc}
\hline Station & $\begin{array}{c}\text { Number } \\
\text { of labour } \\
\text { resources }\end{array}$ & Station & $\begin{array}{c}\text { Number of } \\
\text { labour } \\
\text { resources }\end{array}$ \\
\hline Side Lumbers & 1 & Exterior Wall Panel Framing & 2 \\
Openings & 1 & Special Shape Interior Walls & 1 \\
Interior Wall Framing & 2 & Exterior Wall Drywall and Poly & 2 \\
\hline
\end{tabular}

\subsection{Simulation Model}

The current-state model representing the construction setup described is constructed, and its reliability tested by comparing the outputs from the model to the actual measurements obtained from the plant operating with the modelled layout. The current- 
state model also provides the basis for comparing changes in the future-state models to see how different proposed improvements will affect the entire wall production line.

The current-state model is run for one year and, since it is a stochastic model, multiple runs are used (500 runs). The main model creates the house orders at an interarrival rate of 5 , and with 50 house orders per month. Each house is then defined as either a mini or modular model. The other characteristics, summarized in Table 4-2, are then set. The layout of the main part of the simulation model can be seen in Figure 4-1.

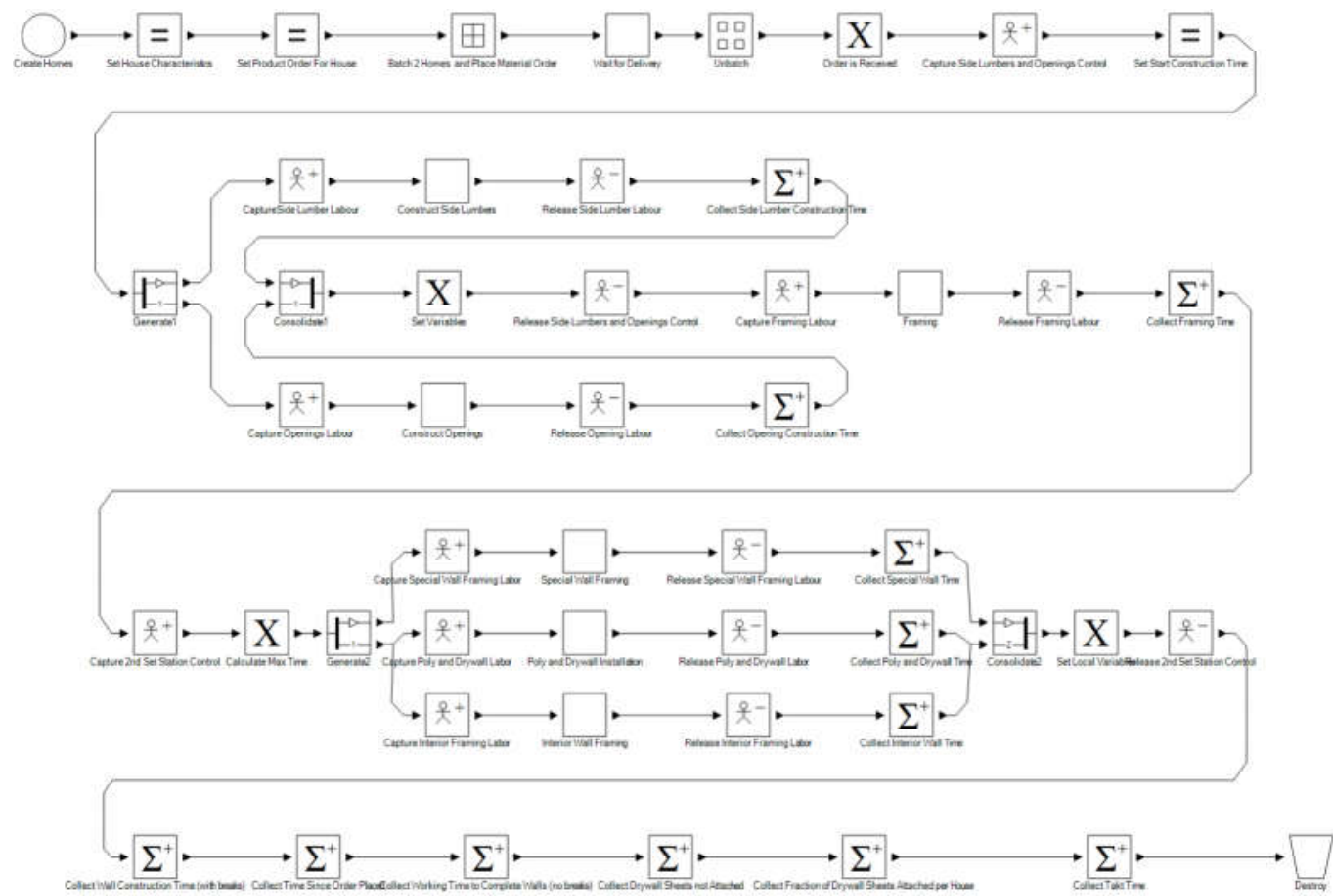

Figure 4-1: Current-state wall panel simulation model

Table 4-2: Variables used in the simulation model

\begin{tabular}{cc}
\hline Characteristic Variables & Time Variables \\
\hline length of the home & time to construct side lumbers \\
number of doors & time to construct openings \\
no. of windows (small, medium, and large) & time to complete exterior wall framing \\
type of home & time to construct special walls \\
wall height & time to install poly and some drywall \\
\hline
\end{tabular}

Each station is simulated so that the required labour is reserved during the time for the task, and released when done. The duration of the task is then calculated depending on the distributions and assumptions in relation to the characteristics of the house, as described above. Priorities are assigned for the labour based on which tasks are most important, as determined through consultation with experts at the plant. The current- 
state simulation results are presented in comparison with the future-state simulation model in the following section.

\subsection{Future-State Simulation Model}

\subsubsection{Labour Change}

To decrease the task times of the exterior wall framing and opening component construction stations, the first change to the simulation model is to add another labour resource to each of these stations in order to increase the rate at which work can be completed. The labour at the exterior wall framing station is increased to three workers, and the labour at the openings construction station is increased to two. It is assumed that the task time, not including setup time, will decrease in proportion to the increase in assigned labour resources, i.e., if the labour resources are doubled, the task time will be halved. The statistics from running the simulation model with the proposed labour assignment show that the takt time can be reduced from 3.975 hours to 2.941 hours. This improved takt time will allow the line to produce the walls for about 2.55 houses per day; however, the statistics also show that the exterior wall framing station still sets the takt time for the wall production line and, in order to further increase production, the task time needs to be further reduced. Table 4-3 summarizes this approach.

Table 4-3: Statistics from the labour change simulation model (six workers)

\begin{tabular}{ccccc}
\hline \multirow{2}{*}{ Statistic } & \multicolumn{2}{c}{ Current State } & \multicolumn{2}{c}{ Future State } \\
\cline { 2 - 5 } & Mean & Std. & Mean & Std. \\
\hline Opening station construction time (hours) & 2.537 & 0.030 & 1.267 & 0.015 \\
Side lumber station construction time (hours) & 0.218 & 0.006 & 0.218 & 0.006 \\
Exterior wall framing station time (hours) & 3.975 & 0.078 & 2.941 & 0.050 \\
Special shape wall construction time (hours) & 0.465 & 0.081 & 0.474 & 0.080 \\
Interior wall framing station time (hours) & 1.805 & 0.043 & 1.786 & 0.043 \\
Exterior poly and drywall station time (hours) & 1.807 & 0.044 & 1.811 & 0.042 \\
\hline
\end{tabular}

\subsubsection{Equipment Change}

One option to further decrease the task time of the exterior wall framing station is to invest in semi-automated equipment. If the company were to invest in a semi-automated machine for the exterior wall panels, it is estimated based on the simulation results tabulated above that the time required to install each stud could be reduced from two minutes to thirty seconds, and that the labour requirement at this station could be reduced by one worker. The statistics found by running the simulation model with these changes show that the takt time can be reduced further to an average of 1.857 hours, which equates to four houses' walls produced per day, and would ensure that the plant can achieve the targeted average production. A summary of this approach can be found in Table 4-4.

Both of the two approaches are recommended to company management. Given that adding labour resources as a means to improving productivity is reactive and may not yield a desirable result, proactive solutions to eliminate non-value-added activities using 
semi-automation are preferable. On this basis, efforts are also made to implement lean principles in order to upgrade existing processes.

Table 4-4: Statistics from the equipment change simulation model

\begin{tabular}{ccccc}
\hline \multirow{2}{*}{ Statistic } & \multicolumn{2}{c}{ Current State } & \multicolumn{2}{c}{ Future State } \\
\cline { 2 - 5 } & Mean & Std. & Mean & Std. \\
\hline Opening station construction time (hours) & 2.537 & 0.030 & 1.268 & 0.016 \\
Side Lumber Station Construction time (hours) & 0.218 & 0.006 & 0.217 & 0.006 \\
Exterior wall framing station construction time (hours) & 3.975 & 0.078 & 1.640 & 0.023 \\
Special shape wall station construction time (hours) & 0.465 & 0.081 & 0.469 & 0.080 \\
Interior wall framing station time (hours) & 1.805 & 0.043 & 1.796 & 0.045 \\
Exterior poly and drywall station time (hours) & 1.807 & 0.044 & 1.807 & 0.046 \\
Takt time (hours) & 3.975 & 0.078 & 1.857 & 0.043 \\
\hline
\end{tabular}

\subsection{Model Validation and Verification}

This model is verified both subjectively by the authors, as well as through the independent validation and verification approach (IV\&V), as described by Sargent (2007). Individual station times are checked by an independent party familiar with the operations at the manufacturing facility to ensure that they are both feasible and appropriate. Another form of validation applied involves checking the takt time against the expected value. The target takt time at the manufacturing facility for which the model is designed is currently about two homes per day. To meet this target the wall line needs to have a takt time of about three-and-a-half to four hours. As seen in Table 4-3 and Table 4-4 above, the takt time for the current state provided by the model is 3.975 hours. This check serves as verification that the model is producing approximately the same volume as the plant in actual practice.

\section{CONCLUSIONS AND RECOMMENDATIONS}

While some changes to production lines, such as labour reallocations, are easy to make and reversible if they do not produce the expected results, others, such as investing in large equipment or reducing overall labour resources, are not. Simulation allows these changes to be tested before they are made to determine their likely effect on the production line. For the labour change and semi-automated equipment scenarios investigated in this research, it can be seen that the production per day can be increased by $34 \%$ by changing the labour assignment and $79 \%$ by investing in semi-automated equipment for the exterior wall framing station. Since the production goal of the company is to produce the walls for four houses each day, this simulation allows them to see that adding labour to the production line will not achieve this goal. With this information, they can see that investing in equipment to aid in the production of the walls is a better alternative to reach their production goal. This information is important since a common solution in the industry is to add more workers in order to increase production, though this may not be the most effective solution. 
The company has since invested in lasers to decrease the measuring time for locations of wall studs, which is an alternative method to decrease the stud installation time, as recommended by the research team and achieved through the application of semi-automated equipment in this simulation. This shows that the conclusions drawn through this work can be useful for decision support in practice.

An important consideration is that the takt time for modular homes is much longer than for mini homes. To arrive at a more reliable measurement that is less dependent on the type of home, each modular home order could instead be switched to an order for two "boxes", which are closer to the size of one mini home, and the takt time goal and calculations could be based on the time per box and not per home. Also, as the data used in this simulation was based on expert opinion and since triangular distributions were used, the accuracy of the model could be improved if a detailed time study of the activities were to be carried out and if distributions were to be fitted to the sampled data.

In future research, analysis should be carried out to further balance the production line, such as tests to determine whether more value can be added to the walls at the stations with extremely short task times, in order to decrease the task times for downstream stations.

\section{REFERENCES}

AbouRizk and Mohamed, 2001. Simphony: An integrated environment for construction simulation in Proceedings of the 2000 Winter Simulation Conference, Orlando, FL, USA, Dec 10-13, pp. 1907-1914.

McLean, C., and Leong, S., 2001. The expanding Role of Simulation in Future Manufacturing in Proceedings of the 2001 Winter Simulation Conference, Arlington, VA, USA, Dec 9-12, pp. 1478-1486.

Moghadam, M., Al-Hussein, M., Al-Jibouri, S., Telyas, A., 2012. Post Simulation Visualization Model for Effective Scheduling of Modular Building Construction. Canadian Journal of Civil Engineering, 39(9), 1053-1061.

Moghadam, M., Lean-Mod: An Approach to Modular Construction Production Efficiency Improvement. Ph.D. Thesis, Department of Civil and Environmental Engineering, University of Alberta, Edmonton, Alberta.

Sargent, R., 2007. Verification and Validation of Simulation Models in Proceedings of the 2007 Winter Simulation Conference, Washington, DC, USA, Dec. 9-12, pp. 124-137.

Shafai, L., 2012. Simulation Based Process Flow Improvement for Wood Framing Home Building Production Lines. M.Sc. Thesis, Department of Civil and Environmental Engineering, University of Alberta, Edmonton, Alberta.

Yu H., Al-Hussein M., Al-Jibouri S., Telyas A., 2013. Lean Transformation in a Modular Building Company: A Case for Implementation. Journal of Management in Engineering, 29(1), 103-111. 